\title{
Some Remarks About Factors of Graphs
}

\section{José R. Correa ${ }^{1}$ and Martín Matamala ${ }^{2}$}

${ }^{1}$ SCHOOL OF BUSINESS UNIVERSIDAD ADOLFO IBÁNEEZ

SANTIAGO, CHILE

E-mail:correa@uai.cl

2DEPARTAMENTO DE INGENIERIA MATEMÁTICA AND CENTRO DE MODELAMIENTO MATEMÁTICO, UMR 2071-CNRS, UNIVERSIDAD DE CHILE, SANTIAGO, CHILE E-mail:mmatamal@dim.uchile.cl

Received December 23, 2005; Revised May 17, 2007

Published online 7 January 2008 in Wiley InterScience(www.interscience.wiley.com).

DOI 10.1002/jgt.20284

Abstract: $A(g, f)$-factor of a graph is a subset $F$ of $E$ such that for all $v \in V$, $g(v) \leq \operatorname{deg}_{F}(v) \leq f(v)$. Lovasz gave a necessary and sufficient condition for the existence of a $(g, f)$-factor. We extend, to the case of edge-weighted graphs, a result of Kano and Saito who showed that if $g(v)<\lambda \operatorname{deg}_{E}(v)<$ $f(v)$ for any $\lambda \in[0,1]$, then a $(g, f)$-factor always exist. In addition, we use results of Anstee to provide new necessary and sufficient conditions for the existence of a $(g, f)$-factor. () 2008 Wiley Periodicals, Inc. J Graph Theory 57: 265-274, 2008

Keywords: graph theory; factors

\section{INTRODUCTION}

A classic problem in graph theory and theoretical computer science is that of finding subgraphs of a given graph with prescribed vertex degrees. Subgraphs

Contract grant sponsor: CONICYT; Contract grant numbers: Anillo en Redes ACT08, FONDECYT 1050710, FONDECYT 1050638.

Journal of Graph Theory

(c) 2008 Wiley Periodicals, Inc. 
of prescribed vertex degrees are commonly referred to as factors. Given a graph $G=(V, E)$ and two integer valued functions defined on $V, g$, and $f$, such that $g(v) \leq f(v)$ for all $v \in V$, a $(g, f)$-factor of a graph $G=(V, E)$ is a spanning subgraph $G^{\prime}=(V, F)$ such that $g(v) \leq \operatorname{deg}_{F}(v) \leq f(v)$, for all $v \in V$. Here, $\operatorname{deg}_{F}(v)$ denotes the number of edges in $F$ incident to $v$.

Lovász [13] gave necessary and sufficient conditions for the existence of $(g, f)$ factors, generalizing the classic results of Tutte $[17,18]$. In particular, he used this characterization to prove that if $G=(V, E)$ has maximum degree $k$, then $E$ can be partitioned into two subsets $E_{1}$ and $E_{2}$, where $\operatorname{deg}_{E_{i}}(v) \leq k_{i}, i=1,2$, for any $k_{1}, k_{2}$ with $k_{1}+k_{2}=k+1$. A special case was shown by Tutte [19], who proved that a $k$-regular graph has a subgraph in which all vertices have degree $r$ or $r+1$, for any $r<k$. Thomassen [16] extended the latter results by proving that if in a graph every vertex has degree $k$ or $k+1$, then there is a subgraph in which every vertex has degree $r$ or $r+1$, for any $r<k$. A "dual" result was obtained by Gupta [6]: If $G=(V, E)$ has minimum degree $k$ and $k_{1}+k_{2}=k-1$, then $E$ can be partitioned into $E_{1}$ and $E_{2}$ such that $\operatorname{deg}_{E_{i}}(v) \geq k_{i}$ for all $v \in V$. Interestingly Heinrich et al. [7] gave a fairly simple necessary and sufficient condition for the existence of $(g, f)$ factor, if $g<f$. This result was extended by Anstee [1,2], who showed that the latter condition is equivalent to the existence of a fractional $(g, f)$-factor, which turns out to be equivalent to the existence of a $(g, f)$-factor with a very special structure. The reader is referred to [14] for an excellent recent survey.

An interesting special class of factors is what we call $\lambda$-factors: Given a graph $G=(V, E)$ and a real number $0<\lambda<1$, a spanning subgraph $G^{\prime}=(V, F)$ is a $\lambda$-factor of $G$ if $\left|\operatorname{deg}_{F}(v)-\lambda \operatorname{deg}_{E}(v)\right| \leq 1$, for all $v \in V$. Extending König's Theorem [12], Hoffman [10] showed that every bipartite graph has a $\lambda$-factor, for each $\lambda$, with $0<\lambda<1$. More precisely, he proved something slightly stronger: If $G=(V, E)$ is bipartite and $0<\lambda<1$, then there is a subset $F \subseteq E$ such that

$$
\left\lfloor\lambda \operatorname{deg}_{E}(v)\right\rfloor \leq \operatorname{deg}_{F}(v) \leq\left\lceil\lambda \operatorname{deg}_{E}(v)\right\rceil, \quad \text { for all } v \in V .
$$

Schrijver noted that this result can be derived using network flows (see p. 361 in [15]). It is natural to ask whether the property just mentioned extends to general graphs. Unfortunately, the answer is negative. To see this, consider an odd cycle and take $\lambda=0.5$. In this case Condition (1) asks for a perfect matching, which does not exist. However, if Condition (1) is replaced by the slightly weaker condition $\left|\operatorname{deg}_{F}(v)-\lambda \operatorname{deg}_{E}(v)\right| \leq 1$, then the result holds for general multigraphs. As noted by Kano and Saito [11], this result can be obtained using the characterizations in [13]. Furthermore, Kano and Saito's result generalizes a number of related results $[6,16,19]$.

\section{A. Our Results}

It is very often the case that graph theoretic concepts become useful in computer science when edge weights are allowed. Therefore, in Section 2 we deal with $\lambda$ - 
factors of edge-weighted graphs. Our first main result of the article says that for any edge-weighted graph there is a factor whose deviation at any vertex does not surpass the maximum weight of an edge adjacent to such vertex. Certainly, the latter result generalizes Kano and Saito's. In Section 3, we go back to study factors of graphs. Our second main result in the article is an alternative necessary and sufficient condition for the existence of a $(g, f)$-factor. Additionally, we derive a simple linear algebra technique that allows us to shorten some arguments and provide a simplified proof of a result of Anstee [1] concerning fractional $(g, f)$-factors.

All results in the article hold for multigraphs. For simplicity in the notation we have chosen to present them in the more restricted framework of graphs without multiple edges.

\section{B. Preliminaries}

Throughout this article $G=(V, E)$ denotes a graph. If $H$ is a subgraph of $G, E(H)$ and $V(H)$ denote its edge set and vertex set, respectively. Moreover, for $F \subseteq E$ and $A \subseteq V, E_{F}(A)$ is the set of edges in $F$ with both ends in $A$. We denote by $\delta(A)$ the set of edges with exactly one end in $A$. Also, $\delta(v)$ denotes the set of edges adjacent to $v$, while if $A \subset V, \delta_{A}(v)=\{v u \in E: u \in A\}$. For a vector $x \in \mathbb{R}^{M}$ and $N \subseteq M, x(N)=\sum_{e \in N} x_{e}$. Similarly, for a function $f: V \rightarrow \mathbb{N}$ and $A \subseteq V$, $f(A)=\sum_{v \in A} f(v)$. As usual, for $F \subset E, \operatorname{deg}_{F}(v)$ denotes the number of edges in $F$ incident to $v$ and deg is the integer valued function assigning to each vertex its degree. Although we defined a factor as a spanning subgraph $(V, F)$ of $G$, we will usually refer to a factor as just the edge set $F$ or its corresponding incidence vector $x \in\{0,1\}^{E}$. On the other hand, we say that a vector $x \in[0,1]^{E}$ such that for every $v \in V, x(\delta(v)) \in[g(v), f(v)]$ is a fractional $(g, f)$-factor of $G$. A half-integral $(g, f)$-factor of $G$ is a fractional $(g, f)$-factor in the set $\{0,1 / 2,1\}^{E}$. If $T \subseteq V, \bar{T}$ will denote the complement of $T$ with respect to $V$. Finally, $|x|_{+}=\max \{0, x\}$ denotes the positive part of $x$.

The following simple lemma will be a useful tool in what follows. For a graph $G=(V, E)$, let $A_{G} \in \mathbb{R}^{|V|} \times \mathbb{R}^{|E|}$ denote its incidence matrix and let $\operatorname{ker}(A)$ be the null space of a matrix $A$.

Lemma 1. For any graph $G, \operatorname{ker}\left(A_{G}\right)=\{0\}$ if and only if each connected component of $G$ is a tree or contains a single cycle, which is odd.

Proof. It is well known that $\operatorname{dim}\left(\operatorname{ker}\left(A_{G}\right)\right)=|E|-|V|+\left|C_{0}\right|$, where $C_{0}$ is the set of bipartite connected components of $G$ (see e.g. [5]). We have that

$$
|E| \geq \sum_{C \in C_{0}}(|C|-1)+\sum_{C \notin C_{0}}|C|=|V|-\left|C_{0}\right| .
$$

Clearly, $\operatorname{ker}\left(A_{G}\right)=\{0\}$ if and only if the previous inequality holds with equality. This happens if and only if each bipartite component is a tree and each nonbipartite component contains exactly one cycle. As the latter are nonbipartite, such a cycle is odd. 


\section{2. $\lambda$-FACTORS OF EDGE-WEIGHTED GRAPHS}

In this section, we extend the concept of $\lambda$-factor to graphs having weights on edges. Our main result essentially says that this more general object always exists. However, the techniques from [11] do not seem to apply in this setting. Specifically our result is the following.

Theorem 2. Let $G=(V, E)$ be a graph, let $w: E \rightarrow[0, \infty)$ and let $\lambda \in(0,1)$. Then, there exists $F \subseteq E$ such that

$$
\left|\sum_{e \in \delta(v) \cap F} w_{e}-\lambda \sum_{e \in \delta(v)} w_{e}\right| \leq \max \left\{w_{e}: e \in \delta(v)\right\}, \quad \text { for all } v \in V .
$$

Note that for $w=\mathbf{1}$, we recover Kano and Saito's result about $\lambda$-factors. We prove this result at the end of this section; the following lemma will be crucial for the proof.

Lemma 3. Let $G=(V, E)$ be a graph and let $l, u: E \rightarrow \mathbb{R}$ be such that $l_{e} \leq 0 \leq$ $u_{e}$ for all $e \in E$. Then, there exists $x \in \prod_{e \in E}\left\{l_{e}, u_{e}\right\}$ such that

$$
\left|\sum_{e \in \delta(v)} x_{e}\right| \leq M(v), \quad \text { for all } v \in V
$$

where $M(v):=\max \left\{u_{e}-l_{e}: e \in \delta(v)\right\}$.

Proof. Consider the set $\Omega$ of all vectors $x \in \prod_{e \in E}\left[l_{e}, u_{e}\right]$ satisfying the following two conditions.

(i) $|x(\delta(v))| \leq M(v)$ for all $v \in V$.

(ii) For every connected component $\alpha$ of the graph $G_{x}=\left(V, E_{x}\right):=(V,\{e \in$ $\left.\left.E: x_{e} \in\left(l_{e}, u_{e}\right)\right\}\right)$, there exists $r \in V(\alpha)$ such that $x(\delta(v))=0$, for each $v \in$ $V(\alpha)-\{r\}$. In this case, we say that $\alpha$ is $x$-rooted at $r$ and that $r$ is a $x$-root of $\alpha$.

Clearly, $\Omega \neq \emptyset$ since $\mathbf{0} \in \mathbb{R}^{E}$ satisfies the conditions. Thus, let $x \in \Omega$ be such that $\left|E_{x}\right|$ is minimum. We first observe that $\operatorname{ker}\left(A_{G_{x}}\right)=\{0\}$. Indeed, assume for a contradiction that there exists $y \in \mathbb{R}^{E_{x}}$ with at least one nonzero coordinate which satisfies $A_{G_{x}} y=0$. Then, if $\hat{y}_{e}=y_{e}$ for $e \in E_{x}$ and $\hat{y}_{e}=0$ for $e \in E-E_{x}$, for any real $\varepsilon$ and for all $v \in V, x(\delta(v))+\varepsilon \hat{y}(\delta(v))=x(\delta(v))$. Thus, one can choose $\varepsilon$ so that $x^{\prime}=x+\varepsilon \hat{y}$ belongs to $\Omega$ and $\left|E_{x^{\prime}}\right|<\left|E_{x}\right|$. Therefore, Lemma 1 implies that if $\alpha$ is a connected component of $G_{x}$, it contains at most one cycle (which has to be of odd length).

To finish the proof it is enough to show that $E_{x}=\emptyset$. For sake of contradiction let us assume that there is a connected component $\alpha$ of $G_{x}$, with $|V(\alpha)| \geq 2$ and $x$-rooted at $r \in V(\alpha)$. First, note that $r$ has to belong to a cycle in $E_{x}$. Otherwise we Journal of Graph Theory DOI 10.1002/jgt 
can take an edge $f=\left(r, r^{\prime}\right) \in E_{x}$ and set $x_{e}^{\prime}=x_{e}$ for edges different from $f$ and $x_{f}^{\prime}=l_{f}$ if $x(\delta(r)) \geq 0$ or $x_{f}^{\prime}=u_{f}$ if $x(\delta(r))<0$. Clearly, $x^{\prime}$ will satisfy (i) and (ii), and $\left|E_{x^{\prime}}\right|<\left|E_{x}\right|$, contradicting the choice of $x$.

Hence, we can assume that there is a cycle $C$ in $E_{x}$, of odd length, containing $r$. Let $e_{1}, \ldots, e_{2 k+1}$ be the edges of $C$, where $e_{1}$ and $e_{2 k+1}$ are the edges incident to $r$. We will again define a vector $x^{\prime}$ which contradicts the choice of $x$. For all edges in $e \in E-E(C)$ we set $x_{e}^{\prime}=x_{e}$. To see how to define the values $x_{e}^{\prime}$ for $e \in E(C)$ consider $\mu^{-}:=l_{e_{1}}+l_{e_{2 k+1}}-x_{e_{1}}-x_{e_{2 k+1}}$ and $\mu^{+}:=u_{e_{1}}+u_{e_{2 k+1}}-x_{e_{1}}$ $-x_{e_{2 k+1}}$. Then, $\mu^{+}-\mu^{-}=u_{e_{1}}+u_{e_{2 k+1}}-l_{e_{1}}-l_{e_{2 k+1}} \leq 2 M(r)$ implying that either $\mu^{+}+x(\delta(r)) \leq M(r)$ or $\mu^{-}+x(\delta(r)) \geq-M(r)$. Thus, for $e \in E(C)$ we set:

$$
\begin{aligned}
& x_{e_{1}}^{\prime}=l_{e_{1}}, x_{e_{2}}^{\prime}=u_{e_{2}}, \ldots, x_{2 k}^{\prime}=u_{2 k}, x_{e_{2 k+1}}^{\prime}=l_{e_{2 k+1}}, \text { if } \mu^{-}+x(\delta(r)) \geq-M(r), \\
& x_{e_{1}}^{\prime}=u_{e_{1}}, x_{e_{2}}^{\prime}=l_{e_{2}}, \ldots, x_{2 k}^{\prime}=l_{2 k}, x_{e_{2 k+1}}^{\prime}=u_{e_{2 k+1}}, \text { if } \mu^{+}+x(\delta(r)) \leq M(r) .
\end{aligned}
$$

With these definitions, $x_{e}^{\prime}=x_{e} \in\left[l_{e}, u_{e}\right]$, for every $e \in E-E(C)$ and $x_{e}^{\prime} \in$ $\left\{l_{e}, u_{e}\right\}$ for every $e \in E(C)$. Hence, $x^{\prime} \in \prod_{e \in E}\left[l_{e}, u_{e}\right]$ and $\left|E_{x^{\prime}}\right|<\left|E_{x}\right|$. To get a contradiction, we show that $x^{\prime} \in \Omega$. To see (i) first note that $x^{\prime}(\delta(v))=x(\delta(v))$ for all $v \in V-V(C)$. Moreover, for every $v \in V(C), v \neq r$, we have $x^{\prime}(\delta(v))=$ $x(\delta(v))+u_{e}+l_{e^{\prime}}-x_{e}-x_{e^{\prime}}$, where $e$ and $e^{\prime}$ are the edges incident with $v$ in $C$. Since $x(\delta(v))=0$ and $\left|u_{e}+l_{e^{\prime}}-x_{e}-x_{e^{\prime}}\right|<M(v)$, it follows that $\left|x^{\prime}(\delta(v))\right|<M(v)$. Since $\mu^{+}>0$ and $\mu^{-}<0$ we also conclude that $\left|x^{\prime}(\delta(r))\right| \leq M(r)$. Therefore, for every $v \in V,\left|x^{\prime}(\delta(v))\right| \leq M(v)$. To see (ii), observe that if $v$ and $v^{\prime}$ are two vertices of $C$ they cannot belong to the same connected component of $G_{x^{\prime}}$, as otherwise there would be an even length cycle in $G_{x}$. Thus, the vertices in $C$ are the $x^{\prime}$-roots of the corresponding connected components of $G_{x^{\prime}}$.

Corollary 4. Let $G=(V, E)$ be a graph and let $l^{\prime}, u^{\prime}: E \rightarrow \mathbb{R}$. For every $x \in$ $\prod\left[l_{e}^{\prime}, u_{e}^{\prime}\right]$ there exists $z \in \prod\left\{l_{e}^{\prime}, u_{e}^{\prime}\right\}$ such that

$$
\forall v \in V,|z(\delta(v))-x(\delta(v))| \leq \max \left\{u_{e}^{\prime}-l_{e}^{\prime}: e \in \delta(v)\right\}
$$

Proof. Let $l_{e}=l_{e}^{\prime}-x_{e}$ and $u_{e}=u_{e}^{\prime}-x_{e}$. From Lemma 3, there is $x_{e}^{\prime} \in$ $\prod_{e \in E}\left\{l_{e}^{\prime}-x_{e}, u_{e}^{\prime}-x_{e}\right\}$ such that $\left|x^{\prime}(\delta(v))\right| \leq \max \left\{u_{e}^{\prime}-l_{e}^{\prime}: e \in \delta(v)\right\}$. Let $z:=x+$ $x^{\prime}$, then, for every edge $e, z_{e} \in\left\{l_{e}^{\prime}, u_{e}^{\prime}\right\}$ and $z(\delta(v))-x(\delta(v))=x^{\prime}(\delta(v))$. Hence $|z(\delta(v))-x(\delta(v))| \leq \max \left\{u_{e}^{\prime}-l_{e}^{\prime}: e \in \delta(v)\right\}$.

Note that Corollary 4 is related to matrix rounding. Indeed, it implies that given a matrix and an interval for each position, one can round each coefficient to one the extremes of its corresponding interval, so that the row and column sums are almost preserved. Furthermore, Corollary 4 extends a graph rounding result by Hell et al. [9, Corollary 3.7] to the case of general bounds on the edges.

With the last corollary at hand, the proof of Theorem 2 becomes remarkably simple. Indeed, let $l_{e}^{\prime}=0, u_{e}^{\prime}=w_{e}$ and $x_{e}=\lambda w_{e}$. From Corollary 4 , there is $z \in$ $\prod_{e \in E}\left\{0, w_{e}\right\}$ such that $|z(\delta(v))-\lambda w(\delta(v))| \leq \max \left\{w_{e}: e \in \delta(v)\right\}$. By defining $F=$ $\left\{e: z_{e}=w_{e}\right\}$ the result is obtained. 


\section{A NEW CHARACTERIZATION FOR THE EXISTENCE OF $(g, f)$-FACTORS}

In this section, we give a new necessary and sufficient condition for the existence of $(g, f)$-factors. This condition find its roots in that of Heinrich et al. [7] and Anstee [1]. Indeed, Heinrich et al. proved that if $g<f$ or $G$ is bipartite a $(g, f)$-factor exists if and only if

$$
\forall A \subseteq V, \quad 0 \leq \Gamma(g, f, A),
$$

where $\Gamma(g, f, A):=f(A)-\sum_{v \in \bar{A}}\left|g(v)-\operatorname{deg}_{\bar{A}}(v)\right|_{+}$.

On the other hand, using the algorithmic approach in [2], Anstee [1] obtained the following results.

Lemma 5 (Anstee [1]). Let $G=(V, E)$ a graph and let $f, g \in \mathbb{Z}^{V}$. Then $G$ has a fractional $(g, f)$-factor if and only if Condition (2) holds.

Theorem 6 (Anstee [1]). A graph $G=(V, E)$ and $f, g \in \mathbb{Z}^{V}$ satisfy Condition (2) if and only if there exists a half-integral $(g, f)$-factor $x$ such that $\left\{e \in E: x_{e}=1 / 2\right\}$ is a vertex disjoint collection of odd cycles contained in the graph induced by $\{v \in V: g(v)=f(v)\}$.

Although Condition (2) is not enough to ensure the existence of a $(g, f)$-factor, Lemma 5 provides good intuition about how much more is needed. In fact, not too much!

Let $F \subseteq E$ and $A \subseteq V$, clearly $2\left|E_{F}(A)\right|=\left(\sum_{v \in A} \operatorname{deg}_{F}(v)\right)-|\delta(A) \cap F|$. Note that if $F$ is a $(g, f)$-factor then, $\sum_{v \in A} \operatorname{deg}_{F}(v) \leq f(A)$ and $|\delta(A) \cap F| \geq$ $\sum_{v \in \bar{A}}\left|g(v)-\operatorname{deg}_{\bar{A}}(v)\right|_{+}$. Therefore,

$$
\forall A \subseteq V, \quad\left|E_{F}(A)\right| \leq\left\lfloor\frac{1}{2}\left(f(A)-\sum_{v \in \bar{A}}\left|g(v)-\operatorname{deg}_{\bar{A}}(v)\right|_{+}\right)\right\rfloor .
$$

Observe that if $F$ is a $(g, f)$-factor then its complement $H:=E-F$ is a $\left(\operatorname{deg}_{E}-f, \operatorname{deg}_{E}-g\right.$ ) factor. Hence,

$$
\forall B \subseteq V, \quad\left|E_{H}(B)\right| \leq\left\lfloor\frac{1}{2} \Gamma(\operatorname{deg}-f, \operatorname{deg}-g, B)\right\rfloor .
$$

Since $\left|E_{F}(A)\right| \geq\left|E_{F}(A \cap B)\right|,\left|E_{H}(B)\right| \geq\left|E_{H}(A \cap B)\right|$, and $\left|E_{H}(A \cap B)\right|+$ $\left|E_{F}(A \cap B)\right|=|E(A \cap B)|$ we obtain the following necessary condition:

$$
\forall A, B \subseteq V, \quad|E(A \cap B)| \leq\left\lfloor\frac{1}{2} \Gamma(g, f, A)\right\rfloor+\left\lfloor\frac{1}{2} \Gamma(\operatorname{deg}-f, \operatorname{deg}-g, B)\right\rfloor .
$$

In the sequel, we will prove that Condition (3) is also sufficient for the existence of $(g, f)$-factors. Interestingly, Condition (3) is stronger than Condition (2), and Journal of Graph Theory DOI 10.1002/jgt 
therefore implies the existence of $(g, f)$-factors in the cases covered by [7]. Indeed, taking $B=\emptyset$ in Condition (3) we obtain $\Gamma(\operatorname{deg}-f, \operatorname{deg}-g, B)=0=|E(A \cap B)|$.

Theorem 7. Let $G=(V, E)$ be a graph and let $f, g$ be integers value functions on $V$. Then $G$ admits a $(g, f)$-factor if and only if it satisfies (3).

Proof. We have already established the necessity. To prove the sufficiency let $x \in[0,1]^{E}$ be a half-integer $(g, f)$-factor (as in Theorem 5) minimizing $\left|E_{x}\right|$, where $E_{x}=\left\{e \in E: 0<x_{e}<1\right\}$. For sake of contradiction let us assume that $x$ is not a $(g, f)$-factor. Then there is an odd cycle $C$ such that $x_{e}=\frac{1}{2}$ for every $e \in E(C)$. For $i=0,1$ let $A_{i}$ be the set of vertices $v$ for which there is an alternating path $P$ of length at least one from $v$ to $C$ such that the edge $e$ of $P$ incident to $v$ satisfies $x_{e}=i$ (here alternating means that if $e$ and $f$ are adjacent then either $x_{e}=1$ and $x_{f}=0$ or $x_{e}=0$ and $\left.x_{f}=1\right)$.

Let $A=A_{0} \cup C$ and $B=A_{1} \cup C$. Therefore the following properties hold: (i) $\forall u \in A, x(\delta(u))=f(u)$; (ii) $\forall u \in B, x(\delta(u))=g(u)$; (iii) $\forall u v \in E, u \in A, v \notin B$ implies $x_{u v}=0$; (iv) $\forall u v \in E, u \in B, v \notin A$ implies $x_{u v}=1$. Properties (i) and (ii) follow from the choice of $x$ (otherwise a half-integer factor $x^{\prime}$ with $\left|E_{x^{\prime}}\right|<\left|E_{x}\right|$ exists). Also, for $u v \in E$ if $u \in A, v \notin C$ and $x_{u v}=1$, then $v \in A_{1}$. Similarly, if $u \in B, v \notin C$ and $x_{u v}=0$, then $v \in A_{0}$. This proves (iii) and (iv). Thus,

$$
\begin{aligned}
\sum_{v \in \bar{A}}\left|g(v)-\operatorname{deg}_{\bar{A}}(v)\right|_{+} & \geq \sum_{v \in B-A}\left(g(v)-\operatorname{deg}_{\bar{A}}(v)\right), \\
& =\sum_{v \in B-A}\left(g(v)-x\left(\delta_{\bar{A}}(v)\right)\right), \\
& =\sum_{v \in B-A} x\left(\delta_{A}(v)\right), \\
& =\sum_{v \in B} x\left(\delta_{A}(v)\right)-\sum_{v \in A \cap B} x\left(\delta_{A}(v)\right), \\
& =\sum_{w \in A} x(\delta(w))-2 x(E(A \cap B)), \\
& =f(A)-2 x(E(A \cap B)) .
\end{aligned}
$$

The first inequality is trivial. From Property (iv) we have that $\operatorname{deg}_{\bar{A}}(v)=x\left(\delta_{\bar{A}}(v)\right)$ which justifies the second step. The third equality is direct from Property (ii). The fourth equality is easy while the fifth follows from Property (iii). The sixth equality comes from Property (i). Therefore $\Gamma(g, f, A) \leq 2 x(E(A \cap B))$. As $2 x(E(A \cap B))$ is odd we get

$$
\left\lfloor\frac{1}{2} \Gamma(g, f, A)\right\rfloor<x(E(A \cap B)) .
$$


Similarly, we can prove that

$$
\left\lfloor\frac{1}{2} \Gamma(\operatorname{deg}-f, \operatorname{deg}-g, B)\right\rfloor<y(E(A \cap B)),
$$

where $y_{e}=1-x_{e}$, for $e \in E$. We finally obtain

$$
\left\lfloor\frac{1}{2} \Gamma(\operatorname{deg}-f, \operatorname{deg}-g, B)\right\rfloor+\left\lfloor\frac{1}{2} \Gamma(g, f, A)\right\rfloor<|E(A \cap B)| .
$$

Although our proof of the last theorem is algorithmic, we do not know whether Condition (3) yields a faster algorithm to find a $(g, f)$-factor. Anstee [2] propose an algorithm that find a $(g, f)$-factor in time $O\left(|V|^{3}\right)$. This running time was improved to $O(\sqrt{f(V)}|E|)$ by Gabow [4]. Later, Hell and Kirkpatrick [8] obtained a $O(\sqrt{g(V)}(|E|+g(V)+p|E|$ ) algorithm ( $p$ being the number of vertices with $g(v)=f(v)$ ), for a more general problem.

To complete the picture we give an alternative short proof of Theorem 6 that uses Lemma 1.

Proof of Theorem 6. The "only if" part of the result is direct from Lemma 5. To see the "if" note that Lemma 5 implies that if $G=(V, E)$ and $f, g \in \mathbb{Z}^{V}$ satisfy Condition (2) then there exists a fractional $(g, f)$-factor of $G$. Let $x$ be a fractional $(g, f)$-factor minimizing the number of fractional edges, that is, minimizing the size of $E_{x}:=\left\{e \in E: 0<x_{e}<1\right\}$.

Clearly we can assume $x(\delta(v))$ is integer for all $v \in V$. Otherwise, we may inductively find a path $e_{1} \ldots e_{k}$ between two vertices with fractional $x(\delta(v))$ such that $x_{e_{i}}$ is fractional along the whole path. Such a path can be perturbed to obtain a fractional factor with either one less fractional edge or one more vertex with integral $x(\delta(v))$.

To conclude we use Lemma 1 . Let $G_{x}=\left(V, E_{x}\right)$ and observe that $\operatorname{ker}\left(A_{G_{x}}\right)=\{0\}$. Indeed, assume for a contradiction that there exist $y \in \mathbb{R}^{E_{x}}$ with at least one nonzero coordinate which satisfies $A_{G_{x}} y=0$. Then, if $\hat{y}_{e}=y_{e}$ for $e \in E_{x}$ and $\hat{y}_{e}=0$ for $e \in E-E_{x}$, for any real $\varepsilon$ and for all $v \in V, x(\delta(v))+\varepsilon \hat{y}(\delta(v))=x(\delta(v))$. Thus, one can choose $\varepsilon$ so that $x^{\prime}=x+\varepsilon \hat{y}$ is a fractional factor with $\left|E_{x^{\prime}}\right|<\left|E_{x}\right|$. Lemma 1 implies that the connected components of $G_{x}$ contain at most one cycle (which has to be of odd length). Now, since $x(\delta(v))$ is integer, a connected component of $G_{x}$ cannot contain vertices of degree 1 . This implies that $G_{x}$ consists of vertex disjoint odd cycles, and again the integrality of $x(\delta(v))$ implies that $x_{e}=1 / 2$ along all those cycles. Finally, by minimality of $\left|E_{x}\right|$, it is straightforward to see that vertices $v$ in such cycles satisfy $g(v)=f(v)$.

\section{AN OPEN PROBLEM}

Finally, we mention an interesting possible extension for the existence of $\lambda$-factors. Let $G=(V, E)$ be a graph and let $\lambda_{1}, \ldots, \lambda_{k} \in(0,1)$ be such that $\sum_{i=1}^{k} \lambda_{i}=1$. Can Journal of Graph Theory DOI 10.1002/jgt 
$E$ be partitioned into $F_{1}, \ldots, F_{k}$ such that for all $v \in V:\left|\operatorname{deg}_{F_{i}}(v)-\lambda_{i} \operatorname{deg}_{E}(v)\right| \leq$ 1 ? Combining Kano and Saito's result with ideas in the proof of Theorem 4 in [3], we can show that the weaker condition $\left|\operatorname{deg}_{F_{i}}(v)-\lambda_{i} \operatorname{deg}_{E}(v)\right|<3$ can indeed be imposed.

\section{ACKNOWLEDGMENTS}

We thank Jácint Szabó and an anonymous referee for useful comments on an earlier version of the article. This work was partially supported by CONICYT through grants Anillo en Redes ACT08, FONDECYT 1050710, and FONDECYT 1050638 .

\section{REFERENCES}

[1] R. P. Anstee, Simplified existence theorems for $(g, f)$-factors, Discrete Appl Math 27 (1990), 29-38.

[2] R. P. Anstee, An algorithmic proof of Tutte's $f$-factor theorem, J Algorithms 6 (1985), 112-131.

[3] J. R. Correa and M. X. Goemans, An approximate König theorem for edge coloring weighted bipartite graphs, SIAM J Comput 37 (2007), 870-894.

[4] H. N. Gabow, An efficient reduction technique for degree-constrained subgraph and bidirected network flow problems, Proceedings of the Fifteenth Annual ACM Symposium on Theory of Computing (STOC), 448-456, 1983.

[5] C. Godsil and G. Royle, Algebraic Graph Theory, Springer-Verlag, New York, 2001.

[6] R. P. Gupta, An edge coloring theorem for bipartite graphs with applications, Discrete Math 23 (1978), 229-233.

[7] K. Heinrich, P. Hell, D. Kirkpatrick, and G. Liu, A simple existence criterion for $(g<f)$-factors, Discrete Math 85 (1990), 313-317.

[8] P. Hell and D. G. Kirkpatrick, Algorithms for degree constrained graph factors of minimum deficiency, J Algorithms 14 (1993), 115-138.

[9] P. Hell, D. Kirkpatrick, and B. Li, Rounding in symmetric matrices and undirected graphs, Discrete Appl Math 70 (1996), 1-21.

[10] A. J. Hoffman, Generalization of a theorem of König, J Wash Acad Sci 46 (1956), 211-212.

[11] M. Kano and A. Saito, [a, b]-factors of graphs, Discrete Math 47 (1983), $113-116$.

[12] D. König, Uber Graphen und ihre Anwendung auf determinantentheorie und Mengenlehre, Math Ann 77 (1916), 453-465.

[13] L. Lovász, Subgraphs with prescribed valencies, J Comb Theory (B) 8 (1970), 391-416. 
[14] M. D. Plummer, Graph factors and factorization: 1985-2003: A survey, Discrete Math 307 (2007), 791-821.

[15] A. Schrijver, Combinatorial Optimization: Polyhedra and Efficiency, SpringerVerlag, New York, 2003.

[16] C. Thomassen, A remark on the factor theorems of Lovász and Tutte, J Graph Theory 5 (1980), 441-442.

[17] W. T. Tutte, The factorization of linear graphs, J Lond Math Soc 22 (1947), $107-111$.

[18] W. T. Tutte. The factors of graphs, Can J Math 4 (1952), 314-328.

[19] W. T. Tutte, The subgraph problem, Ann Discrete Math 3 (1978), 289-295. 